\title{
R-EDEEC: Reserved-Enhanced Distributed Energy Efficient Clustering Routing Protocol for Wireless Sensor Networks
}

\author{
Anamika Chauhan \\ Dept. of CSE \\ Delhi Technological University \\ New Delhi, India
}

\author{
S. Indu \\ Professor \\ Dept. of EC \\ Delhi Technological University \\ New Delhi, India
}

\begin{abstract}
In the recent years a widespread increase in application of Wireless sensor networks (WSN) has been seen, Consequently many Protocols have been developed to enhance the performance of WSN. The earliest approach was a homogeneous clustering based homogenous protocol Low energy adaptive clustering hierarchical (LEACH). But with variety of increasing applications many protocols with variations and improvements have been developed. In this paper we have studied the heterogeneous protocol, DEEC, its enhanced Version Enhanced (EDEEC) and suggested an improvement. This paper proposes a new routing protocol "Reserved Enhanced distributed energy efficient clustering (R-EDEEC) routing protocol for WSN". In this protocol, the concept of 'Reserved' node is introduced to improve the efficiency by increasing the lifetime and stability of the network. The proposed approach successfully shows better performance in comparison to LEACH and DEEC.
\end{abstract}

\section{General Terms}

Sensor networks, energy efficiency

\section{Keywords}

\section{LEACH , EDEEC, DEEC, WSN}

\section{INTRODUCTION}

Wireless Sensor Network (WSN) are ad-hoc networks, they are composed of a set of wireless sensors. The major constraint for wireless sensor network is energy for sustenance and operation. As mostly the sensor node is battery operated battery and therefore has limitation of energy. Sometimes the battery can be recharged, or may be have only one time life. Also they are usually deployed in remote and hostile places and hence lifetime of WSN is limited. To be able to optimise the performance of the network therefore energy consumption must be optimised. The energy cost is twofold, one for transmission and processing of data and two for operation and maintenance of network itself. To prolong and optimise the network lifetime, several simultaneous software and hardware based approaches may be used. Including design of efficient routing protocols and sensors with high energy besides designing low-power sensor nodes. The challenge in the ongoing research is to develop lowpower communication with low cost on-node processing and self-organized connectivity/protocols. Several protocols were developed to make the communication energy-effective to prolong the life of the networks. These protocols were different in how they improve the communication and transmission of the packets in the network but they all based on clustering approach in the network. The homogeneous protocol(due to same initial energies of all nodes) LEACH[1] which considered all nodes of same energy used the probabilistic approach to elect the cluster head and the probability of selection of a node as the cluster head decides after how many rounds a node can be again cluster head. But this approach does not guarantee for a high energy node to be cluster head. After homogenous, a heterogeneous protocol came, SEP[4] which divided the network into two kind of nodes having different energy normal nodes and higher energy nodes called advanced nodes. The probabilistic method of selecting the cluster heads varies for these two types of nodes. These probabilities are based on their energies. This was done to improve the stability period (the time when first node dies [4]) of the network and to extend the life. Several modified versions of SEP routing schemes were proposed such as ESEP, ZSEP etc. ESEP [3] increase the heterogeneity to level 3 using the normal, intermediate and advanced nodes and these were taken in the order of increasing initial energies resp. ZSEP[3] divided the network in the form of zones which resemble clusters so that proper distribution of the nodes and energies in each cluster can be done These successfully outperformed the SEP but shortcoming of these protocols including SEP of not considering the effect of residual energies on the cluster head selection probabilities for different kind of nodes. Another Protocol, DEEC [2] took the concept of residual energy of a node and average energy of the network in electing the cluster heads with existing heterogeneity of the nodes and successfully improved the routing in network. Several versions of DEEC were explored which improved it further such as DDEEC and EDEEC. DDEEC [2] estimate the average overall energy in the network and $\mathrm{CH}$ is selection probability is based on each nodes residual energy as used in DEEC. DDEEC takes the same concept and adds threshold residual energy and when energy level of advanced and normal nodes goes below the threshold residual energy then both kind of nodes (normal and advanced ) have equal probability of being selected as $\mathrm{CH}$. EDEEC [2], an enhancement of DEEC proposed to add one more level of heterogeneity in the network (super node) which increased the lifetime as well. It has been evaluated in [2] that DDEEC has low stability period, lifetime and throughput as compared to the EDEEC. In this paper we worked on EDEEC, and our protocol R-EDEEC successfully improved the stability period and throughput of the network which ultimately enhanced the life of the network. This paper is divided as II section is about related research section III is about the proposed protocol REDEEC and section IV and V. about simulation and results and conclusion.

\section{RESEARCH BACKGROUND}

Low-Energy Adaptive Clustering Hierarchy (LEACH) Low- 
energy adaptive clustering hierarchy, is a routing protocol for heterogeneous networks. LEACH protocol focuses mainly on increasing the performance based on three key points as follows:

- Increased life time of network.

- Lower consumption and improved energy efficiency

- Hierarchical information aggregation mechanisms reduces redundancy

LEACH is a called hierarchical because it partitions the network into a set of smaller sub units called clusters. Instead of each node sending information directly to the base station , the data is collected by the cluster head, it is aggregated and compressed and then forwarded to the base station. The aggregated data is received by the base station which in single hop from the $\mathrm{CH}$. The data collection is performed periodically by the cluster, using the Time division multiple access (TDMA). TDMA, each member node transmits data in the slot allocated to it eliminating inference among cluster members. Also to eliminate inter-cluster interferences in the network code division multiple access (CDMA) is used among clusters. Since it is hierarchical, operation of LEACH is performed in the following two phased manner:

(i) Set-up phase: in this period initially all the nodes are divided and clusters are formed. This includes cluster head advertisement and creation of schedules for TDMA and CDMA.

(ii) Steady-state phase: the transmission of data to the base station is done in this phase after aggregation and compression .

The process of Cluster head selection in the LEACH is performed as follows:

LEACH uses a probabilistic method for calculating a value called threshold. This threshold is calculated using the probability of cluster heads selection and round number. This threshold is necessary to calculate the node eligibility to become a cluster head based on $1 / p$. A random number $g$, the value of which lies in $[0,1]$ set. Based on the threshold value and $\mathrm{g}$ corresponding node for which the threshold is calculated become cluster head otherwise not

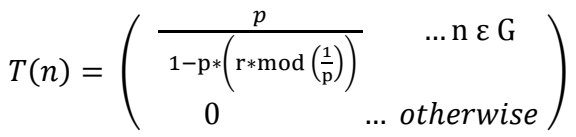

For ensuring fresh candidate nodes in each round $\mathrm{CH}$ will be selected from nodes that not been $\mathrm{CH}$ in the last $1 / \mathrm{p}$ rounds will not be elected in this round. They are represented by variable $\mathrm{G}$.

This completes the set-up phase now steady-state phase starts. It is seen that LEACH perform well and is comparatively energy efficient but suffers from some shortcomings:

- The residual or remaining energy of the nodes is not taken into consideration for calculating the probability of cluster heads selection. Thus even extremely low energy node are selected as a cluster head. This low energy $\mathrm{CH}$ will die soon as it will drain very fast. Since transmission is only between $\mathrm{CH}$ and BS, This will eventually lead to frequent change in $\mathrm{CH}$ and also reduced network .

Also in LEACH cluster formation is random, therefore it suffers with unbalanced clusters. Thus some clusters may die earlier due unequal distribution of the energy.

\section{DISTRIBUTED ENERGY EFFICIENT CLUSTERING (DEEC)}

DEEC overcomes LEACHs shortcoming by introducing heterogeneity. All nodes do not have equal probability. But the probability of being selected $\mathrm{CH}$ is directly proportional to energy of the node

In DEEC heterogeneity is introduced by considering not only the total energy of a node but residual as well as the average energy as a criterion for of the cluster head selection. In DEEC $\mathrm{E}(\mathrm{r})$ is used to denote the mean energy of nodes considered in a $\mathrm{CH}$ selection round

$\overline{\mathrm{E}}(\mathrm{r})=1 / \mathrm{n} \sum_{1}^{\mathrm{n}} \mathrm{E}_{\mathrm{i}}(\mathrm{r}) \quad\{$ for $\mathrm{i}=1$ to $\mathrm{n}\}$

In a network comprising of $n$ nodes. In this $E(r)$ is considered as the reference energy, it shows

$$
\begin{aligned}
& \mathrm{P}_{i}=\mathrm{p}_{\mathrm{opt}}\left[1-\frac{\left(\bar{E}(r)-E_{i}(r)\right)}{E(r)}\right] \\
& =\mathrm{p}_{\text {opt }} \mathrm{E}_{\mathrm{i}}(\mathrm{r}) / \overline{\mathrm{E}}(\mathrm{r})
\end{aligned}
$$

In heterogeneous networks, different nodes have different probabilities of cluster heads selection and different epoch time. In this case since the nodes have been divided into two levels, $p_{\text {opt }}$ is now calculated as the weighted probabilities for each type the low energy normal and high energy advanced nodes as given below

$$
P_{i}=\left(\begin{array}{cc}
\frac{p_{\text {opt }} * E_{i}(r)}{(1+\text { a. } m) * E(r)} & \text {... normal node } \\
\frac{p_{\text {opt }} *(1+\alpha) E_{i}(r)}{(1+a . m) * E(r)} & \ldots \text { advanced node }
\end{array}\right)
$$

Thus using the average energy for evaluation of the threshold. The average energy of the $\mathrm{E}(\mathrm{r})$ of $\mathrm{r}^{\text {th }}$ round is given as follows:

$$
\overline{\mathrm{E}}=1 / n * \mathrm{E}_{\text {total }}(1-r / \mathrm{R})
$$

Where $\mathrm{R}$ denotes the network lifetime. Thus in DEEC all nodes dissipate energy almost at same rate .

Therefore it can be assumed that all the nodes would live almost for same time.

Base station broadcasts the value of total energy and number of. Nodes can calculate average energy of the network, their own residual energy, threshold and accordingly select $\mathrm{CH}$.

\section{ENHANCED DISTRIBUTED ENERGY EFFICIENT CLUSTERING (EDEEC)}

EDEEC extended the heterogeneity level to three level of nodes i.e. normal, intermediate and advanced. Here, the energy of intermediate node is between the normal and advanced nodes. In EDEEC, the nodes and respective energy are distributed according to fractions $\mathrm{m}$ and $\mathrm{m}_{0}$ of total $\mathrm{n}$ as

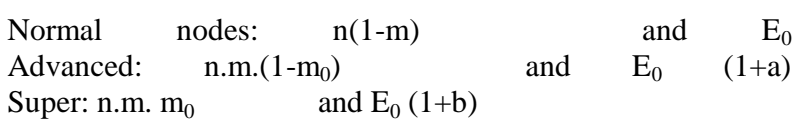




$$
P_{i}\left(\begin{array}{ll}
\frac{p_{o p t} * E_{i}(r)}{\left(1+m\left(a+m_{0} b\right)\right) * E(r)} & \text { normal node } \\
\frac{p_{\text {opt }} * E_{i}(r) *(1+a)}{\left(1+m\left(a+m_{0} b\right)\right) * E(r)} & \text { advanced node } \\
\frac{p_{o p t} * E_{i}(r) *(1+b)}{\left(1+m\left(a+m_{0} b\right)\right) * E(r)} & \text { super node }
\end{array}\right)
$$

EDEEC used the same mechanism for estimating the energy in the network as proposed in DEEC. Since the probabilities calculated depend on the average of the network at round r.This average energy is estimated as

$$
\overline{\mathrm{E}}(r)=1 / n * \mathrm{E}_{\text {total }}(1-r / \mathrm{R})
$$

Where $\mathrm{R}$ is calculated using DEEC protocol.

\section{PROPOSED APPROACH}

The proposed approach is to minimize energy consumption in the process of Cluster head selection .The selection of $\mathrm{CH}$ is done again and again after the end of each round in protocols like LEACH, DEEC, EDEEC etc. This consumes a lot of energy of the network. To minimize this energy consumption in the selection process, we introduce the concept of 'Reserved' node. A reserved node is selected in each iteration (cycle) along with the Cluster head. The reserved node is the node having second highest energy in the cluster.

During first half of the cycle cluster head and reserved node are selected and for the first round reserved node is made to sleep. So the reserve node remains inactive and hence don't transmit any data to the cluster head. Thus, there is minimal consumption of energy in the reserved node, just to keep it alive. After a round is over the reserved node becomes the new cluster head without any kind of overhead in calculating threshold values to determine who will become the next cluster head and hence saving a considerable amount of energy and thus increasing overall lifetime of the network. After the end of the second half of the cycle, new cycle is started and the cluster head and the reserved node are selected again as done before and this keeps on happening till all nodes die. We observe that the use of reserved node increases the throughput, stability and overall lifetime of the network. So nodes probability of eligibility to become cluster head and energy in our scheme are

Normal nodes: $\mathrm{n}(1-\mathrm{m})$ and $\mathrm{E}_{0}$

Advanced: n.m. $\left(1-\mathrm{m}_{0}\right)$ and $\mathrm{E}_{0}(1+\mathrm{a})$

Super: $\mathrm{n} . \mathrm{m} . \mathrm{m}_{0} \quad$ and $\mathrm{E}_{0}(1+\mathrm{b})$

Where $a=1 / 2 ; b=1$;

Here $\mathrm{p}$ is the probability of choosing the cluster heads in the network so a node becomes eligible for cluster head again after $1 / p_{0}$. So no of cluster heads should be $n^{*} p_{0}$ if $n$ is the total no of nodes. In our scheme nodes are distributed according to constant $\mathrm{m}$ and $\mathrm{m}_{0}$.

We have not introduced any kind of new node which have a different energy level so probabilities and threshold are calculated as done in EDEEC.

\section{FLOWCHART}

This flowchart is showing the starting of the network and then cluster head and reserved node are elected. After node-cluster head communication is done, the reserved node becomes the new cluster head and node-cluster head communication is done again. This finishes the second round and the process continues until there are no alive nodes left.

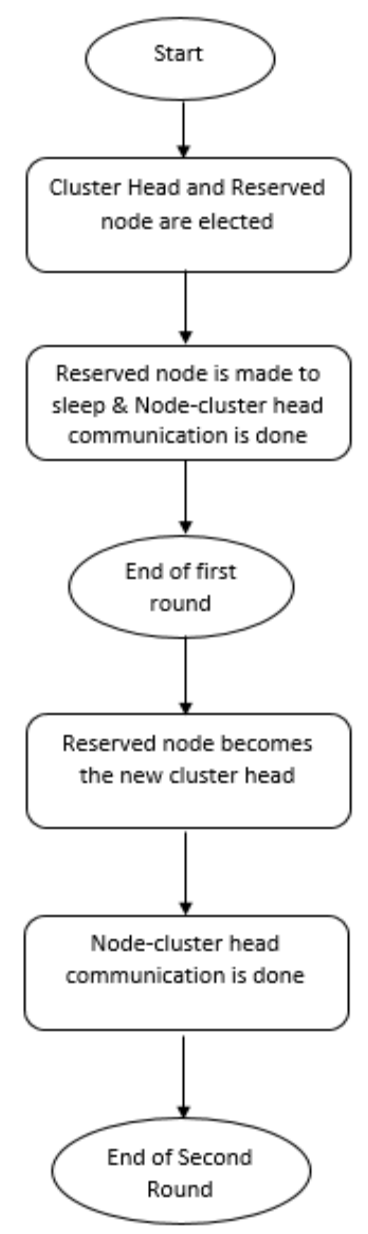

Fig. 1 Flowchart of the protocol

\section{SIMULATION AND RESULTS}

This project is simulated in MATLAB software and C++ programming language. We have compared the performance of four protocol LEACH, DEEC , EDEEC and our approach i.e. Reserved enhanced distributed energy efficient clustering (R-EDEEC) routing protocol by estimation based on four performance measures. The positions are calculated on the basis of manual deployment of the nodes so all nodes mutually know positions of each other in the network. The various parameters used for simulation of the network are listed in Table 1.

Table 1. List of simulation parameters

\begin{tabular}{|l|l|}
\hline Parameters & Value \\
\hline Area & $100 * 100$ square meters \\
\hline Base station & $(50,50)$ (in m) \\
\hline Initial Energy & $0.1 \mathrm{~J}$ \\
\hline Transmission Energy & $50 \mathrm{~nJ} / \mathrm{bit}$ \\
\hline Receiver Energy & $50 \mathrm{~nJ} / \mathrm{bit}$ \\
\hline No Of Nodes & 100 \\
\hline Free Space Amp Energy & $10 \mathrm{pJ} / \mathrm{bit}^{\mathrm{m}} / \mathrm{m}^{2}$ \\
\hline $\begin{array}{l}\text { Multi path Amplification } \\
\text { Energy }\end{array}$ & $0.0013 \mathrm{pJ} / \mathrm{bit} / \mathrm{m}^{4}$ \\
\hline
\end{tabular}




\begin{tabular}{|l|l|}
\hline Message Size(B) & 4000 bits \\
\hline Round & 1000 \\
\hline Aggregation Energy & $5 \mathrm{nj} / \mathrm{bit} /$ packet \\
\hline $\mathrm{p}_{\mathrm{opt}}$ & 0.04 \\
\hline $\mathrm{M}$ & 0.5 \\
\hline $\mathrm{m}_{0}$ & 0.5 \\
\hline
\end{tabular}

The performance of the four protocols has been estimated using four performance measures.

1. Alive nodes (or Dead nodes (inverse of alive nodes)) for stationery nodes it also represents network coverage

2. Throughput (number of packets send to the Base Station by per cluster)

3. Stability Period (the round in which first node becomes dead.

4. Network Lifetime It is duration during which network works in successful way and it is max. no of rounds up to at least one node is alive

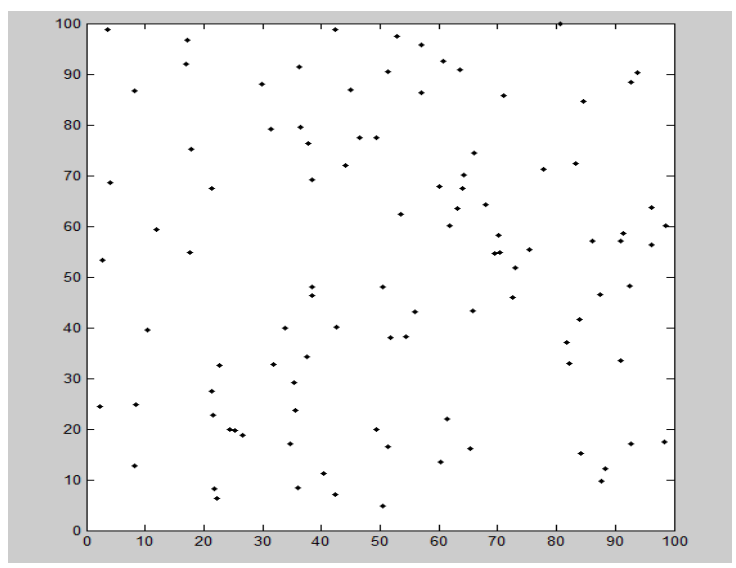

Fig. 2 Distribution of nodes

Figure 1 and 2 shows the distribution of nodes and alive nodes existence respectively in the network with increasing rounds in different protocols LEACH, DEEC, EDEEC and REDEEC. R-EDEEC has maintained longevity of the nodes in the network to large no of rounds.

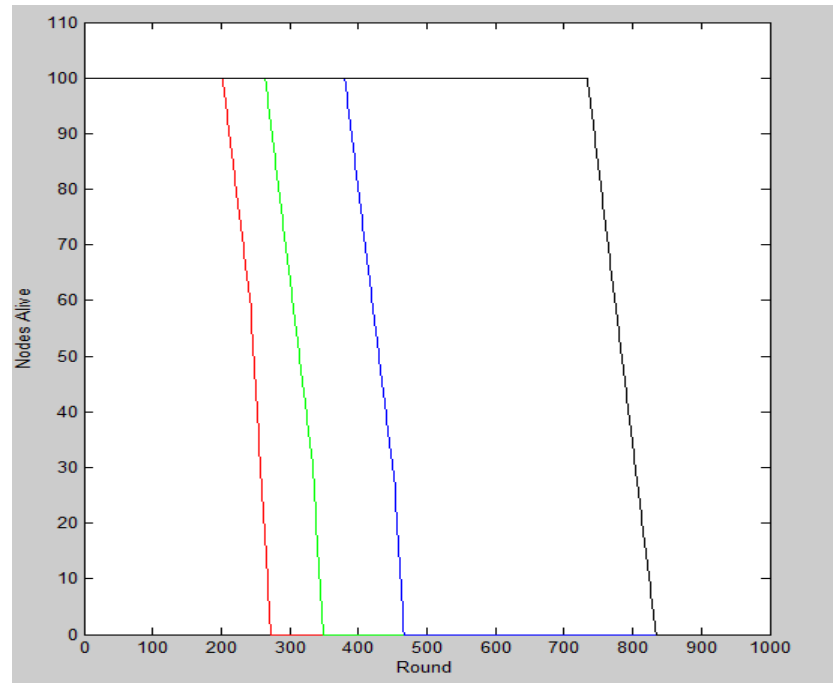

Fig. 3 No of Alive Nodes(Coverage)

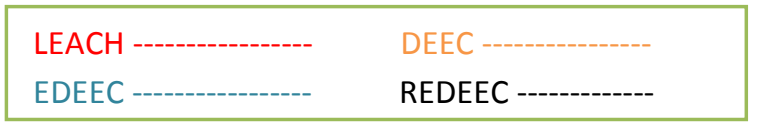

Table 2 has listed the stability period (as stated previously it is the round in which first node dies) for LEACH, DEEC, EDEEC and REDEEC which is clearly stating period is very high for REDEEC. Table 3 and 4 show that lifetime and throughput increased by implementation of REDEEC.

Table 2. Stability period Comparison(number of rounds)

\begin{tabular}{|l|l|l|l|l|}
\hline PROTOCOL & LEACH & DEEC & EDEEC & REDEEC \\
\hline Simulation 1 & 201 & 273 & 384 & 718 \\
\hline Simulation 2 & 203 & 272 & 370 & 630 \\
\hline Simulation 3 & 195 & 254 & 332 & 569 \\
\hline Simulation 4 & 210 & 294 & 379 & 639 \\
\hline
\end{tabular}

Table 3. Network lifetime Comparison

\begin{tabular}{|l|l|l|l|l|}
\hline PROTOCOL & LEACH & DEEC & EDEEC & REDEEC \\
\hline Simulation 1 & 271 & 351 & 486 & 822 \\
\hline Simulation 2 & 243 & 336 & 441 & 735 \\
\hline Simulation 3 & 253 & 346 & 482 & 803 \\
\hline Simulation 4 & 255 & 327 & 450 & 756 \\
\hline
\end{tabular}

Table 4. Throughput for various protocol

\begin{tabular}{|l|l|l|l|l|}
\hline PROTOCOL & LEACH & DEEC & EDEEC & REDEEC \\
\hline Simulation 1 & 257 & 331 & 463 & 738 \\
\hline Simulation 2 & 233 & 324 & 424 & 669 \\
\hline Simulation 3 & 235 & 328 & 463 & 726 \\
\hline Simulation 4 & 246 & 313 & 440 & 689 \\
\hline
\end{tabular}

From figures and tables we can see that REDEEC has outperformed the older three protocols in all parameters such as stability period, lifetime and throughput. 


\section{CONCLUSION}

R-EDEEC improves the energy efficiency of the network by introduction of one more variation of heterogeneity in the flat structure, followed by using hierarchical heterogenety of nodes thus further optimizing the protocol. Introducing the concept of Reserved node has had the following effects :

- $\quad$ Significant increased the stability period

- Reduction in the energy consumption in selecting cluster head by decreasing the number of iterations of cluster head selection

- Increase in lifetime of network by selecting cluster head every second round and thus ultimately reducing energy consumed and improved the throughput of the network.

\section{REFERENCES}

[1] Padmalaya Nayak, Pallavi Shree, "Comparison of Routing Protocols in WSN using NetSimSimulator:
LEACH Vs LEACH-C", in: International Journal of Computer Applications (0975 - 8887) Volume 106 No.11, November 2014

[2] Anamika Chauhan, Amit Kaushik, "TADEEC: Threshold Sensitive Advanced Distributed Energy Efficient Clustering Routing Protocol for Wireless Sensor Networks", in: International Journal of Computer Applications (0975 - 8887) Volume 96 - No.23, June 2014

[3] G. Smaragdakis, I. Matta, A. Bestavros, "SEP: A Stable Election Protocol for clustered heterogeneous wireless sensor network", in: Second International Workshop on Sensor And Actor Network Protocols and Applications (SANPA 2004), 2004

[4] Parul Saini, Ajay K. Sharma, "E-DEEC- Enhanced Distributed Energy Efficient Clustering Scheme for heterogeneous WSN", in: $20101^{\text {st }}$ International Conference on Parallel, Distributed and Grid Computing (PDGC - 2010). 